\title{
Intelligent Astronaut Associate for Next Generation Space Systems
}

\author{
Olu Olofinboba, Michael Dorneich \\ Honeywell Laboratories \\ 3660 Technology Drive, MN65-2600 \\ Minneapolis, MN 55418 \\ (612) 951-7112, (612) 951-7488 \\ olu@ieee.org, michael.dorneich@honeywell.com \\ Kerry Williams \\ Honeywell Defense \& Space Electronic Systems \\ 19019 N. 59th Ave., MS: AZ76-2P12 \\ Glendale, AZ 85308 \\ (602) 822-3453 \\ kerry.williams@honeywell.com
}

\begin{abstract}
Astronaut crews operating next-generation spacecraft and space systems will be tasked with managing highly complex automated systems far from Earth. The distances involved and communications lags present with Earth-based control centers will induce a necessary autonomy never before experienced in Human Spaceflight. Working more autonomously, these astronauts will need to be keenly aware of external situations, internal status and health of spacecraft systems, and options for dealing with faulty equipment and off-nominal situations. Crews will also have to understand how the spacecraft automation is to be used in light of the current mission situation, and how to trade off operational objectives as necessary to use the automation effectively ${ }^{1}$.
\end{abstract}

An Intelligent Astronaut Associate (IAA) has the potential to enhance the ability of astronaut crews to work more efficiently, to cope with uncertainty and to address the unique challenges outlined above. This paper describes an initial implementation of the IAA which would provide an intelligent automation interface to the astronaut crew, dynamically providing relevant real-time information, while monitoring cognitive state and workload to determine how best to present pertinent information to the crew. The IAA would also include knowledge-based reasoning capability, and cockpit technologies such as integrated alerting and notification, presented via a task driven, intuitive, consistent natural language for interfacing with the automation.

\section{TABLE OF CONTENTS}

1. INTRODUCTION .......................................................1

2. ARCHITECTURE OVERVIEW .................................2

3. CONCLUSIONS .....................................................5

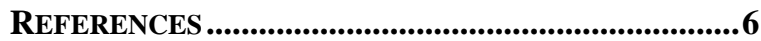

BIOGRAPHY ........................................................................7

${ }^{1}$ 0-7803-8870-4/05/\$20.00@ 2005 IEEE.

\section{INTRODUCTION}

This paper describes an initial implementation of an Intelligent Astronaut Associate (IAA). Future manned space flight will be held to higher safety and efficiency standards, yet astronauts will be required to perform more informationally-dense and cognitively-demanding tasks with larger, more dynamic, and more complex networks of human-automation teams. The confluence of these factors will raise information processing and decision demands on astronauts to unparalleled levels. Intelligent automation and quantum steps in the crew-autonomy interface technology will be critical to meeting these challenges. The IAA builds on recent advances in cognitive gauge development, integrated alerting, mixed-initiative control, human-centered design and embedded training. The IAA will consist of intelligent modules that will assess mission situations, astronaut cognitive state, tasks being performed, overall mission or plan, and other factors relevant to the operator. IAA will adapt this information to provide each crewmember with the right information in the right format at the right time. Figure 1 illustrates a simplified version of the functional architecture for the IAA.

The Cognitive State Assessor (CSA) will maintain awareness of astronaut capabilities using real-time, directlysensed measures of cognitive state. The Context Assessor (CA) will maintain awareness of all aspects of the vehicle, habitat, environment, and crew; and interpret them for pertinence to IAA' crew assistance role. The Mitigation Manager (MM) will use knowledge of current and planned activities, along with the activity needs identified by the Context Assessor, to determine a mitigation strategy for needed activities or activity modifications. It will include an Interface Manager (IM) for proposing modifications to the way information and input capabilities are made available to 
Olofinboba O., Dorneich, M., and Williams, K. (2005) "Intelligent Astronaut Associate," Proceedings of the IEEE Aerospace Conference, Big Sky, MT, March 5-12, 2005.

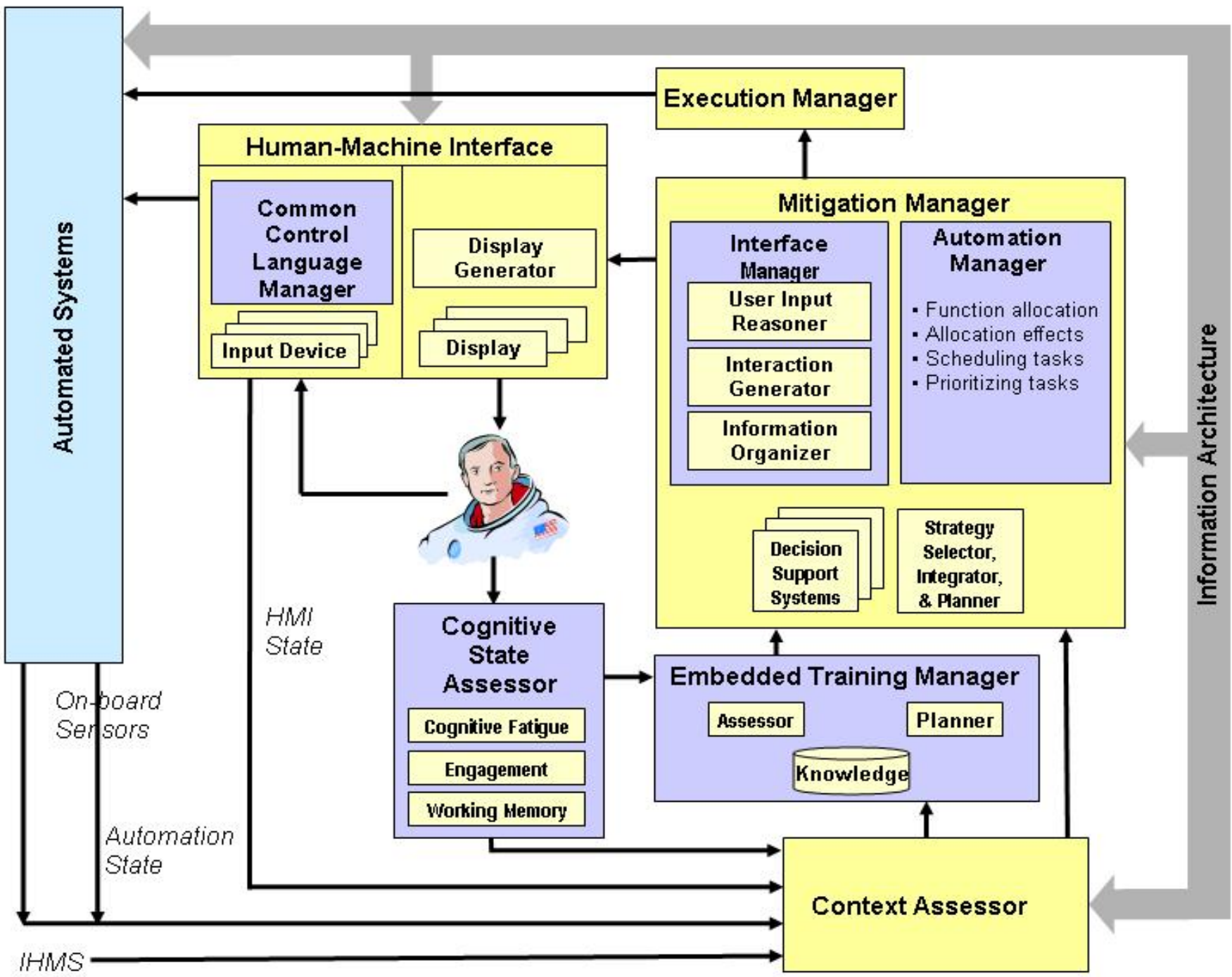

Figure 1 - Simplified system architecture of IAA

the astronaut and an Automation Manager (AM) for proposing mitigation strategies that modify the vehicle or habitat's equipment behaviors to be made by the automation itself (perhaps subject to human approval). The Execution Manager (EM) will ensure that plans created by the MM are realized in the real-world via the Human-Machine Interface (HMI) which includes a Common Control Language (CCL) for natural communications between IAA and the astronauts, and the available automation systems. An Intelligent Embedded Training Manager (IETM) will provide the capability to detect training needs and requests and then formulate and deliver training interventions customized to the context and the crew member. An underlying Information Architecture will support and define communications between modules, the vehicle/habitat's systems, astronauts, and ground crew.

\section{ARCHITECTURE OVERVIEW}

The Intelligent Astronaut Associate implementation illustrated in Figure 1 is a framework architecture tying several enabling modules together.

Specific IAA objectives include:

(1) IAA is aware of the astronaut's cognitive state at all times.

(2) Information presentation is adapted to make the best use of the astronaut's available physical and cognitive resources.

(3) Required information is available anytime, anywhere, in the best available format. Interactions can migrate from large screen monitor to personal digital assistant (PDA) to speech input/output to tactile interaction as needed and available. 
(4) Astronauts can interact with information displays and automation by means of a single, shared and intuitive vocabulary.

(5) Alarm and notification information from disparate systems is integrated, filtered, prioritized, and presented consistently to reduce information processing demands when reacting to off-nominal situations.

(6) Embedded training is available and customized to the individual's context, changing proficiency level, and available cognitive capacity.

\section{Cognitive State Assessor}

The Cognitive State Assessor (CSA) will achieve our goal of awareness of the cognitive state of the astronaut at all the times using real-time, directly sensed measures of cognitive state [2].

CSA will use non-invasive sensors such as electroencephalogram (EEG) and electrocardiogram (ECG). EEG is the gold standard for providing high-resolution spatial and temporal indices of cortical electrical activity from scalp electrodes. ECG is an electrical recording of heart activity. IAA will leverage these non-invasive sensors to provide the raw signal for refined indices that measure operator states of interest. For instance, IAA will exploit an EEG-power measure that is validated as a measure of attention within a multitasking environment [4].

IAA will also evolve neural net approaches for generating cognitive profiles based on multi-dimensional input from the aforementioned indices. Cognitive profiles provide insight into an astronaut's attentional state, working memory load, workload as impacted by fatigue, and autonomic arousal levels. To train a neural net to classify interesting astronaut states, IAA will require domain expert input to identify the intersection of astronaut task demands with hazardous cognitive states.

\section{Context Assessor}

The Context Assessor (CA) will maintain awareness of all aspects of the vehicle, habitat, environment, and crew and interprets them for pertinence to IAA' crew assistance role. It will accept input from four types of sensor fusion and interpretation packages:

(1) Onboard sensors tracking crew and mobile equipment locations, equipment states, etc.

(2) Integrated Health Management System (IHMS) information for reporting vehicle or habitat states and diagnosing or prognosticating problems.

(3) Cognitive State Assessor for awareness of astronauts' cognitive states.
(4) HMI State and Automation Systems State for reporting the current state of the displays and automated systems with which the astronaut is interacting.

These sensed states will be inputs to two reasoning systems: the Activity Interpreter (AI) and the Activity Needs Assessor (ANA). The AI will interpret data from the sensing components to detect and interpret user activities (against a library of known activities). An "activity" is our primary way of representing, tracking and reasoning about user behaviors. Activities are represented as full or partial task models with hierarchical and sequential relationships. Resource requirements (including time, information and human cognitive capacities) can be organized and stored with activity descriptions and, therefore, tracked along with them. Formal procedures are one, but not the only, kind of activity. The AI's functionality is similar to what has been referred to as an Intent Interpreter or Task Tracker. It will provide a concise and dynamic estimate of who is doing what.

The ANA will assess environment, system, and human states more broadly to reduce the torrent of sensor and system data available every second to a narrow stream of explanatory states IAA refer to as situations. Situations can also arise when a known or expected activity is not seen to be occurring as expected or scheduled. As a simple example, knowing that a system needs to be powered down before a procedure can be initiated implies that either a human or an automation process needs to perform the "power off" activity. The output of the ANA is not a recommended activity, per se, but rather the status of current activities and the need for new activities that the Mitigation Manager (and, perhaps other onboard decision aiding systems incorporated in it—see below) will identify or develop. The CA reasoning will result in a set of current activities the crew is engaged in (from the $\mathrm{AI}$ ) and their status, along with a set of activity needs (from the ANA).

\section{Mitigation Manager}

The Mitigation Manager (MM) will use knowledge of current and planned activities along with the activity needs identified by the Context Assessor, to determine a mitigation strategy for activity modifications. The MM consists of four major subcomponents:

(1) An Interface Manager (IM) will propose modifications to the way information and input capabilities are made available to the astronaut. It must maintain an integrated picture of system status and strategic information for predictive situation awareness and planning, while providing specific information pertinent to the activities being worked by each crew member. The IM is itself composed of a User Input Reasoner that determines the necessary input capabilities or affordances the user will need, an Interaction Generator that provides alternate methods 
for presenting the needed interactions on available devices to the user, and an Information Organizer that prioritizes, filters, and clusters incoming information for presentation [3][5].

(2) An Automation Manager (AM) will propose mitigation strategies that modify the vehicle or habitat's equipment behaviors as made by the automation itself (perhaps subject to human approval). There are four broad categories of possible mitigations: (1) task/information scheduling, (2) task offloading, (3) task sharing, and (4) modality management (handled by Interface Manager). The AM will be capable of dynamic, real-time, function allocation: deciding which functions allocated to human and which are allocated to automation (task offloading), and which tasks can be shared between the human and the automation (task sharing). The AM will be capable of determining the effects of function allocation on task-subtask interactions, workload, mission management, and performance under stress. The AM will schedule tasks and manage interruptions by prioritizing tasks to minimize multi-tasking costs, thereby assisting the operator to switch among tasks without losing situational awareness (task scheduling). Our innovative approach calls for a moving middle ground of human-automation interaction through variableinitiative interaction, which can assume more or fewer duties of a shared task depending on the situation. This approach contrasts with most autonomous systems, in which the level of interactions between human and automation is fixed at the design stage [6].

(3) Decision Aiding Systems (DASs) will provide advice in either machine-executable form (to the AM and Strategy Selector, Integrator, and Planner (SSIP)) or in human-executable form (to the IM and SSIP). IAA will be able to interface to other DASs but IAA will also incorporate a specific DAS to support human interaction with procedures.

(4) A Strategy Selector, Integrator and Planner (SSIP) will receive strategies proposed by the other three MM modules, then select and integrate them to generate a plan to be carried out by the Execution Manager. It may also compose a strategy by seeking methods for missing pieces from the above components (e.g., Automation could shut this valve, but only with human permission, hence the AM must deliver a request to the user-the SSIP brokers a request to IM and integrates the resulting plan pieces). Planning portions will also reason about plan feasibility given user, habitat, vehicle, and automation states and capabilities.

\section{Intelligent Embedded Training Manager}

The vast number of spacecraft systems and the broad range of tasks they support present significant training challenges. Infrequent tasks, such as those associated with mission anomalies, and normal procedures that are relevant only rarely in a mission are particularly challenging. Waning proficiency levels during an extended mission can have a major impact on the safety and efficiency of operations. The IAA system will incorporate intelligent embedded tutoring functionality to help flight crews develop and maintain expertise over extended missions. Training will be situated in the actual context of use. Where appropriate, crew members will be able to practice tasks using actual systems that will be used to perform mission tasks. Additionally, trainees will be able to practice mission relevant tasks using software simulations.

The Intelligent Embedded Training Manager (IETM) will detect training needs, and request, formulate, and deliver training interventions customized to context. It will be composed of:

(1) A Training Needs Assessor that uses crew and vehicle state data processed by the Context Assessor to detect the need for training interventions (e.g., rare procedure is scheduled, task performance degrades, or astronaut request),

(2) A Training Mitigation Planner to develop training intervention plans for detected situations, and

(3) Training Knowledge that encapsulates training procedures and mission knowledge.

Training efforts will be guided by intelligent tutoring agents [1]. Like human tutors, intelligent tutoring agents will be able to tailor feedback and guidance based on dynamic assessments of task performance and a student's emotive and cognitive state. Task performance will be assessed using a combination of plan recognition algorithms and cognitive models. This process, known as model tracing [7], will pinpoint a learner's problem solving context within a broad problem space, making it possible to tailor assistance not merely to an individual's background or general temperament, but also to his or her current context and cognitive state. With real-time assessment of cognitive state, the Training Mitigation Planner can modulate workload levels to adapt the pace of the training to current working memory capacity.

\section{Human-Machine Interface}

The Human-Machine Interface (HMI) will realize the interaction design of the Interface Manager outputs by configuring a multi-modal set of devices to display information to the human and allow him or her to input control actions. The HMI will include three principal components:

(1) Display Generator,

(2) Common Control Language (CCL) Manager, and 
(3) Physical input and display devices.

The HMI Design Generator will automatically generate taskrelevant user interfaces that support the coordination of visual, auditory, and tactile communications.

The CCL is a shared vocabulary for facilitating information transfer from the user to the system. When an astronaut learns to operate a complex automated system, he or she must memorize a large set of procedures and operating rules for each system interface. From his/her point of view, the process is often non-intuitive and even arbitrary. An infrequently accessed function can take several minutes of trial and error before the astronaut finds the correct path through the interaction logic. CCL solves this problem by using an operational logic already familiar to astronauts, the content and syntax of spoken language, as the basis for user interface interaction logic. It allows interactions with most automation subsystems based on a familiar subset of natural language, whether spoken, written, or graphical. CCL's taskdriven, intuitive, consistent language for instructing the system will greatly enhance communication between astronauts, automation, and ground controllers [8].

The HMI will be able to operate across a variety of systems and modalities. Display systems include, but are not limited to PDAs, fixed monitors, and aural annunciators. They can operate in a range of modalities (visual, aural, and haptic). Input devices include, but are not limited to cursor control devices, keyboards, control boards, and speech recognition devices. The Interface Manager will be able to change the modality of information presentation, depending on available physical presentation systems, and based on the operator's current cognitive state [9].

\section{Execution Manager}

The Execution Manager (EM) will ensure that the plans created by the Mitigation Manager are realized in the "real world." The EM is composed of: (1) Command Translators that can realize the abstract instructions from the IM and AM on specific devices, (2) a Real-Time Execution Monitor (RTEM) that will detect finer-grained variations in execution performance (especially for real-time correction of automation behaviors that avoid rerunning the whole loop), and (3) an Exception Handler (EH) to provide repair actions for exceptions the RTEM identifies.

\section{Information Architecture}

An underlying Information Architecture (IA) will support and define communications between the other modules, the rest of the vehicle/habitat's systems, and the astronauts and ground crew. The architecture includes:

(1) A "vocabulary," probably in the form of an ontological representation, for the entities, actions and relationships of interest to the IAA system as a whole, and

(2) A means of storing and sharing information expressed in that vocabulary among the IAA modules (e.g., a blackboard, databus, sub-pub ring, etc.)

The strength of the Intelligent Astronaut Associate is in integrating all these modules to provide a completely integrated and superior solution.

\section{Conclusions}

\section{Significance to future exploration system}

IAA will greatly reduce the time spent on training and retraining operators to interact with automated control systems. It will help eliminate operator errors on infrequently accessed functions. These benefits will be critical for maintaining a safe human presence in deep space, where recurrent training to keep operators proficient will be extremely difficult or nonexistent. IAA is applicable to any environment where astronauts interact with automation including extra-vehicular activity, intra-vehicular activity, and robotic collaboration.

\section{Advantages of IAA}

Providing an adaptive and intelligent astronaut automation management system directly affects the overall affordability, flexibility, reliability, and effectiveness of systems.

Closely coupled with integrated system health management systems, IAA will allow human intervention before serious failures, to avoid downtime, costly repairs, or even more expensive replacements. Using a Common Control Language (CCL) and HMI Display Generator design approach also provides lifecycle engineering advantages for significant affordability benefits. It allows for the simple addition of new functions without major changes to controls or training. Infrequently needed functions take up a large part of training time and require the most recurring training. In a CCL-based system, infrequent function command strings are created exactly like all others.

Understanding what the system is going to do when you give it a command reduces erroneous inputs and gives the operator better situation understanding. The IAA cognitive state advances will reduce the cognitive load associated with programming the system, improving the crew's ability to solve problems in high stress situations. These advances will also increase information processing by adapting the work environment in response to the evolving state of the astronaut and detect hazardous cognitive states to minimize incidence or impact of human error. Input from the IAA Cognitive State Assessor will also help task models disambiguate between states. 
Olofinboba O., Dorneich, M., and Williams, K. (2005) "Intelligent Astronaut Associate," Proceedings of the IEEE Aerospace Conference, Big Sky, MT, March 5-12, 2005.

A CCL-based command system is independent of the interface used. This design provides operators with flexibility and redundancy in the way commands are entered. The strings of words can be generated via keyboard, menus, icons and fixed function buttons, knobs, or speech recognition. Similarly, the IAA Interface Manager will be able to change the modality of information presentation, depending on available physical presentation devices and based on the operator's current cognitive state [2][9]. IAA Intelligent Embedded Training technologies will also foster high levels of operational proficiency and flexibility among crew members while substantially reducing training time relative to conventional training approaches.

\section{REFERENCES}

[1] John R. Anderson and Kevin, J. Gluck, "What role do cognitive architectures play in intelligent tutoring systems?" In D. Klahr \& S. M. Carver (Eds.), Cognition \& Instruction: Twenty-five years of progress, 227-262, 2001.

[2] Michael Dorneich, Stephen Whitlow, Patricia M. Ververs, Jim Carciofini, and Janet Creaser, "Closing the Loop of an Adaptive System with Cognitive State," Human Factors and Ergonomics Society Conference Proceedings, September 20-24, 2004.

[3] Michael C. Dorneich., Olu Olofinboba., Patricia M. Ververs, Woodrow Winchester, and Subramani Krishnamurthi, "Alerting and Notification of Conditions Outside the Aircraft (ANCOA): Integrated Alerting Guidelines and Conceptual Design," Technical Report prepared for NASA Ames Research Center under contract NAS1-00107, Honeywell Laboratories, September 30, 2002.

[4] Frederick G. Freeman, Peter J. Mikulka, Lawrence J. Prinzel, and Mark W. Scerbo, "Evaluation of an adaptive automation system using three EEG indices with a visual tracking task," Biological Psychology, 50, 61-76, 1999.

[5] Robert S. McCann, and Jeffrey McCandless, "Humanmachine teaming for dynamic fault management in nextgeneration launch vehicles," Joint Army-Navy-NASA-Air Force 3rd Modeling and Simulation Meeting Proceedings, December 1-5, 2003.

[6] Christopher A. Miller, Harry B. Funk, Michael C. Dorneich, and Stephen Whitlow, "A Playbook Interface for Mixed Initiative Control of Multiple Unmanned Vehicle Teams," Digital Avionics Systems Conference Proceedings, October 27-31, 2002.

[7] John R. Anderson, Albert T. Corbett, Kenneth R. Koedinger, and Ray Pelletier, "Cognitive tutors: Lessons Learned," The Journal of the Learning Sciences, 4, 167207, 1995.

[8] Victor Riley, "Developing a pilot-centered autoflight interface," SAE World Aviation Congress, Warrendale, PA: Society of Automotive Engineers, 2000.

[9] Robin R. Penner and Erik S. Steinmetz, "DIGBE: Adaptive User Interface Automation," AAAI Spring Symposium Proceedings, March 20-22, 2000. 
Olofinboba O., Dorneich, M., and Williams, K. (2005) "Intelligent Astronaut Associate," Proceedings of the IEEE Aerospace Conference, Big Sky, MT, March 5-12, 2005.

\section{BIOGRAPHY}

Olu Olofinboba is a Senior Scientist in the Human Centered

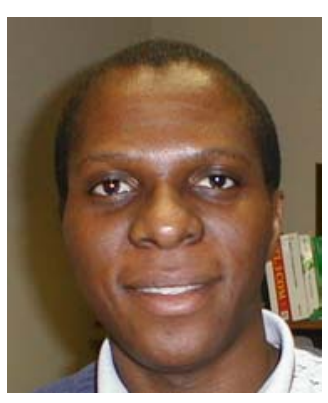

Systems section of Honeywell Laboratories. He has led several external- and Honeywell-funded research and development programs, including the Cockpit Control Language program. As a research scientist in the University of Minnesota's Human Factors Laboratory, he worked primarily on intelligent transportation projects. He obtained his BSEE (Computers) and MS in Computer Engineering from the University of Southern California and is an IEEE Senior Member.

Kerry Williams is a Principal Engineer at Honeywell where

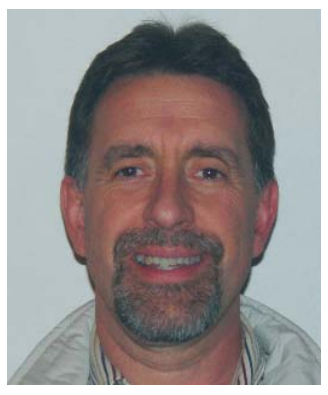

he currently serves as integrated product team (IPT) lead for Human Centered Design for Space Exploration. Mr. Williams has over 20 years experience in design and development of large software systems for space and defense related applications as well as leadership roles in System Engineering and Program Management. He holds a Bachelor of Science degree in Computer Science from Texas Christian University and a Master of Science in Aeronautical Science (with honors) from Embry-Riddle Aeronautical University and is a member of ACM, the IEEE Aerospace and Electronic Systems Society and Computer Society and the American Institute of Aeronautics and Astronautics.

Michael Dorneich is a Senior Scientist in the Human

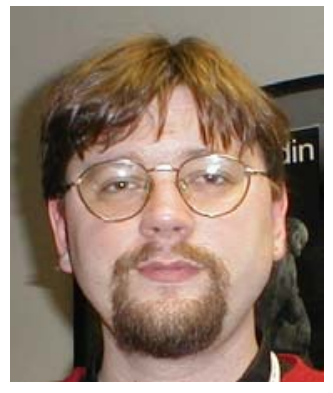
Centered Systems section of Honeywell Laboratories. He has worked NASA-funded programs to design integrated cockpit alerting systems, evaluate integrated weather avoidance route planners, and design cockpit control upset cueing systems. He is co-Principal Investigator on the DARPAfunded Augmented Cognition program and was cognitive engineering lead on Mixed-

Initiative Control of Automata-teams (MICA). His expertise is the design of collaborative systems, adaptive humanmachine interaction, interactive learning environments, and decision-support systems. He obtained his BS and MS in Electrical Engineering and Ph.D. in Industrial Engineering/Human Factors from the University of Illinois. 
Olofinboba O., Dorneich, M., and Williams, K. (2005) "Intelligent Astronaut Associate," Proceedings of the IEEE Aerospace Conference, Big Sky, MT, March 5-12, 2005. 